Western Washington University

Western CEDAR

3-20-2000

\title{
Formation of Uniaxial Molecular Films by Liquid- Crystal Imprinting in a Magnetic Field
}

Joseph D. Mougous

Andrew J. Brackley

Katherine Foland

Robert T. Baker

David L. Patrick

WesternWashington University, david.patrick@wwu.edu

Follow this and additional works at: https://cedar.wwu.edu/chemistry_facpubs

Part of the Chemistry Commons

\section{Recommended Citation}

Mougous, Joseph D.; Brackley, Andrew J.; Foland, Katherine; Baker, Robert T.; and Patrick, David L., "Formation of Uniaxial Molecular Films by Liquid-Crystal Imprinting in a Magnetic Field" (2000). Chemistry Faculty and Staff Publications. 3.

https://cedar.wwu.edu/chemistry_facpubs/3 


\title{
Formation of Uniaxial Molecular Films by Liquid-Crystal Imprinting in a Magnetic Field
}

\author{
Joseph D. Mougous, Andrew J. Brackley, Katherine Foland, Robert T. Baker, and David L. Patrick* \\ Department of Chemistry, Western Washington University, Bellingham, Washington 98225
}

(Received 21 September 1999)

\begin{abstract}
Scanning tunneling microscopy was used to study molecular order in monolayer organic films formed by solution-phase growth from thermotropic liquid crystal solvents. The films develop macroscopically uniaxial alignment, with adlayer orientation controlled by an external magnetic field through interactions mediated by the liquid crystal. Results are presented for two films deposited from nematic and smectic- $A$ solvents, along with a discussion of the alignment mechanism.
\end{abstract}

PACS numbers: 83.70.Jr, 68.45.-v, 81.10.Dn

There is growing interest in the fabrication of highly ordered molecular films for a range of applications, and considerable effort has been invested in the molecular design, synthesis, and characterization of crystalline films with targeted properties [1]. However, a major limitation to constructing useful devices based on molecular materials, and to obtaining a better understanding of the properties of molecular solids, is that most organic compounds of interest yield polycrystalline films with random or partially random domain orientation. Numerous applications, ranging from molecular electronics and photonics to protein crystallography would benefit from a general method for growing films with uniform alignment [2].

Herein we report that macroscopically uniaxial molecular films can be prepared by substituting a thermotropic liquid crystal (LC) solvent for the conventional liquid solvents ordinarily used in solution-phase crystal growth. The solvent's nematic order is imprinted on the film through anisotropic interactions arising from surface anchoring and curvature elasticity. When film growth is carried out in a magnetic field, orientational alignment can be externally controlled.

We studied several monolayer organic films deposited onto graphite substrates from different LC solvent $/ \mathrm{molec}$ ular solute combinations using both nematic and smectic LCs. Results from two systems are presented here: (1) $n$-tetracosanoic acid (TA) deposited from the nematic LC ZLI-1565 [3] (0.3\% by weight) and (2) 4'octyl-4-cyanobiphenyl (8CB) deposited from a neat fluid. $8 \mathrm{CB}$ is a room-temperature smectic- $A$ LC [4]. The second system represents the simplest implementation of the method, in which the LC solvent and solute were identical. Films of TA and 8CB were deposited onto substrates of highly oriented pyrolytic graphite (HOPG ZYH grade, Advanced Ceramics, Inc.) measuring $\sim 1 \mathrm{~cm}^{2}$, which were deeply immersed $(>2 \mathrm{~mm})$ in a reservoir of the $\mathrm{LC} /$ solute mixture. Each system was heated to $\sim 100{ }^{\circ} \mathrm{C}$, then gradually cooled to room temperature in a magnetic field oriented parallel to the substrate plane (Fig. 1). In both systems, a single polycrystalline monolayer formed at the graphite interface in contact with the bulk LC solvent. After cooling, samples were removed from the field and analyzed with scanning tunneling microscopy (STM). The STM tip penetrated through the LC fluid to image molecules in the monolayer at the graphite interface.

Both $8 \mathrm{CB}$ and TA adsorb strongly on graphite, forming commensurate domains oriented along one of three symmetric directions separated by $120^{\circ}[5,6]$. In the absence of a magnetic field, these three directions are energetically equivalent. Since the crystallographic orientation of the substrate varied over micron length scales, samples prepared with no magnetic field developed macroscopically random orientation. However, this symmetry was broken for films deposited within a magnetic field, leading to macroscopically uniaxial order. To measure this uniaxial order, the orientation of adsorbed molecules was assessed

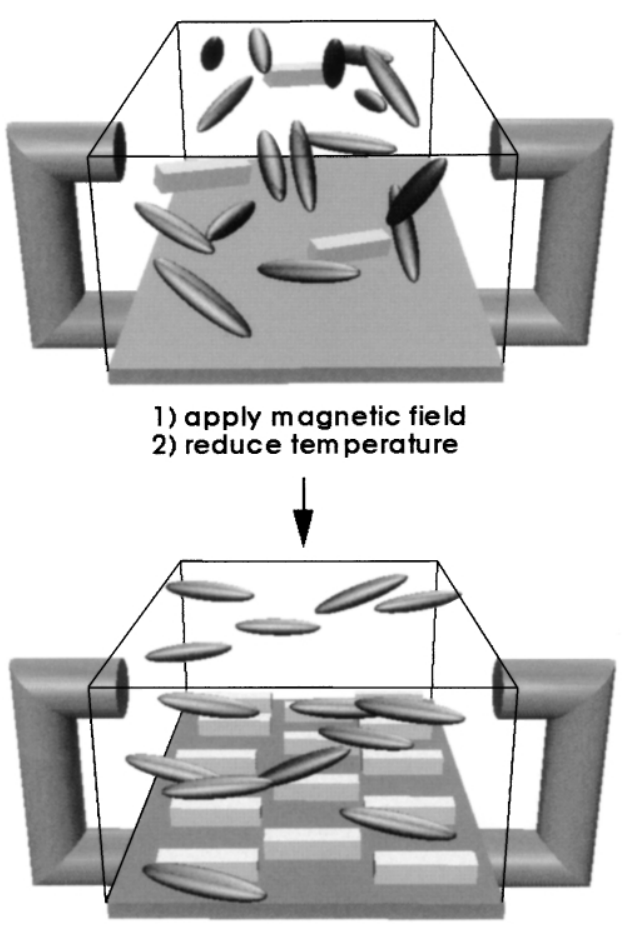

FIG. 1. Samples were prepared by immersing a graphite substrate in fluid mixtures based on thermotropic LC solvents at temperatures above the monolayer melting point. Application of a magnetic field followed by gradual cooling resulted in uniformly aligned polycrystalline films. 
at approximately 200 widely spaced locations across several samples using STM.

Figure 2 presents a histogram showing the experimental distribution (shaded bars) of TA alignment measured for several films prepared in a $12.6 \mathrm{kG}$ field. These films displayed macroscopically uniaxial order controlled by the external field-nematic order had been imprinted on the monolayer. The mean angle between the long molecular axis and the field was $\phi_{0}=12 \pm 5^{\circ}$; the STM image atop the figure shows the most probable domain orientation. To normalize for micron-scale variations in local substrate orientation, we plot $P(\phi)=$ $N(\phi) /\left[N(\phi)+N\left(\phi+120^{\circ}\right)+N\left(\phi-120^{\circ}\right)\right]$, where $N(\phi)$ is the number of domains in which the long molecular axis oriented at an angle $\phi$ with respect to the field. This procedure corrects for statistical undersampling associated with making measurements at a finite number of surface locations and also eliminates any systematic orientational bias which would occur if the distribution of local substrate orientations included in the sampling was not truly random.

In 8CB monolayers, the long molecular axis also oriented approximately parallel to the field. However, in this

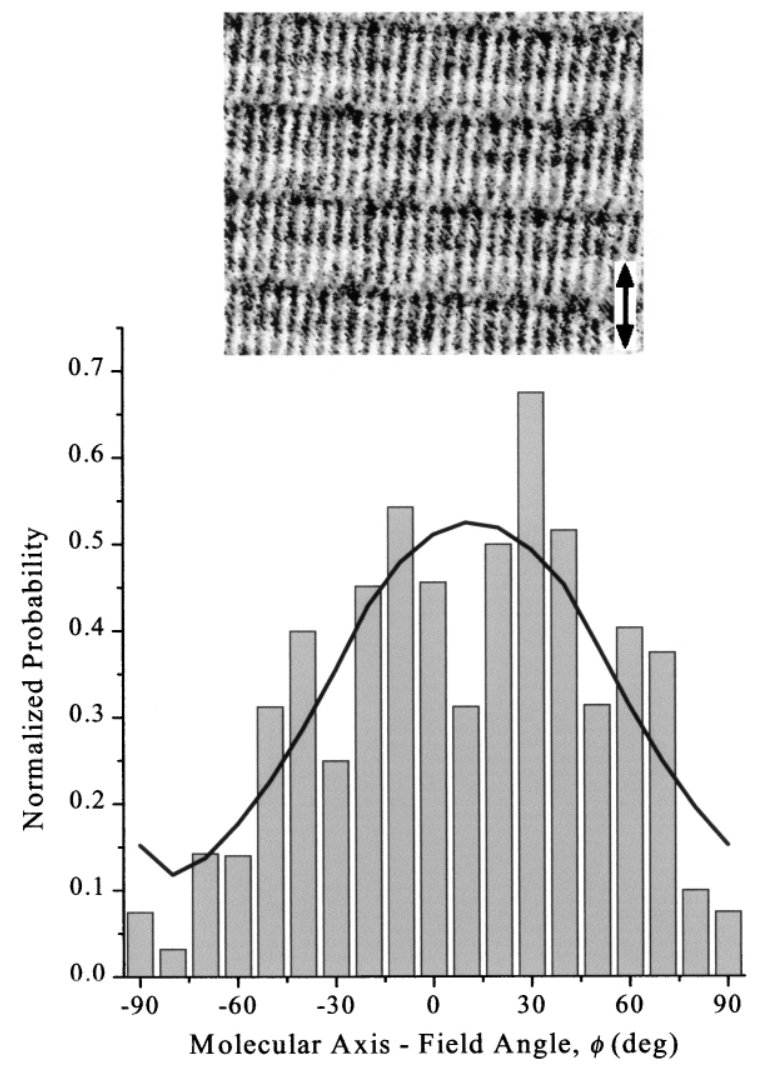

FIG. 2. Alignment in tetracosanoic acid films grown from the LC ZLI-1565. The STM image shows the most common domain orientation, with the field axis indicated by an arrow $\left(I_{\text {tunn }}=\right.$ $\left.1.0 \mathrm{nA}, V_{b}=-1.0 \mathrm{~V}, 10 \times 10 \mathrm{~nm}\right)$. The histogram shows an experimental alignment distribution based on 217 observations from several samples prepared in a $12.6 \mathrm{kG}$ field. The solid line corresponds to the model described in the text. case the distribution is bimodal, because we have plotted the angle between the molecular rows and the field, rather than the angle involving the molecular axis (Fig. 3). For $8 \mathrm{CB}$, row orientation is a more convenient descriptor of overall alignment than the orientation of individual molecules, since each molecular axis in the unit cell points in a slightly different direction, and because the 8CB monolayer is chiral. Chirality develops as a result of molecular adsorption, when rotation about the $\mathrm{C}-\mathrm{C}$ bond linking the cyanobiphenyl headgroup to the alkyl tail group is quenched. This gives the molecule a bow shape, and hence chirality in two dimensions. STM images of leftand right-chiral domains are shown in Fig. 3. Because the molecular axis forms an angle of approximately $\pm 30^{\circ}$ to the rows, and because the sign of this angle depends on domain chirality, there were two favored row orientations, $\phi_{0}= \pm\left(54.6 \pm 8.6^{\circ}\right)$ with respect to the field. When rows oriented in these directions, the molecular axis was essentially parallel to the field.

Figure 3 shows the experimental distribution of $8 \mathrm{CB}$ row alignment in a $7.2 \mathrm{kG}$ field. We prepared films at other field strengths as well (not shown), from 1.2 to $13 \mathrm{kG}$, observing that the quality of orientational order improved with increasing field strength, reaching a limit
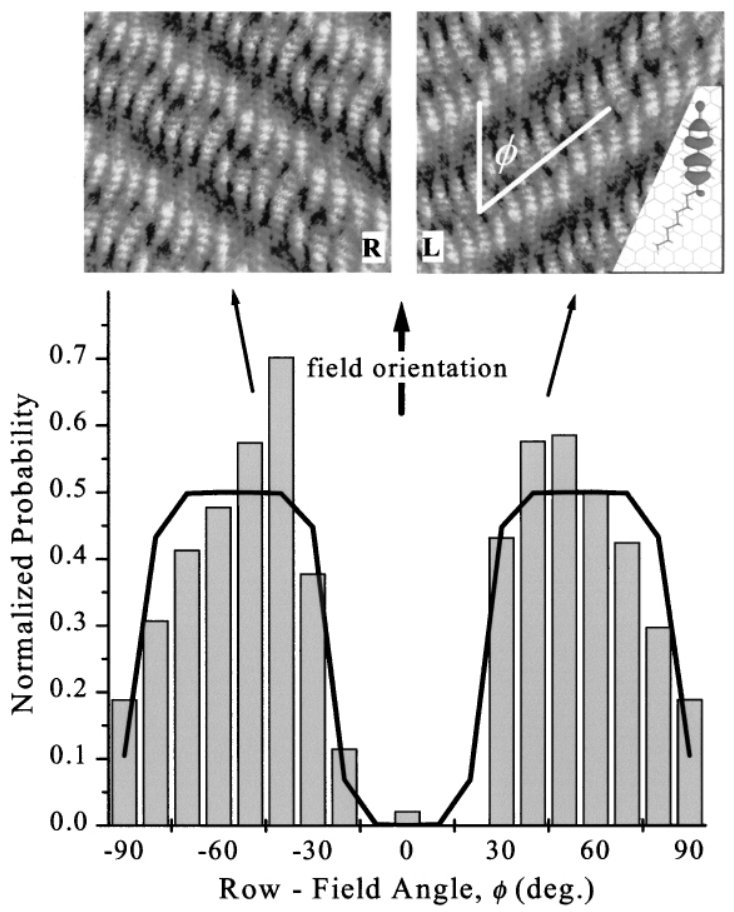

FIG. 3. Top: 8CB forms a crystalline monolayer on graphite with right- $(R)$ and left- $(L)$ handed domains. The images show the preferred orientation of each chirality at high field strength $\left(I_{\text {tunn }}=4.0 \mathrm{nA}, V_{b}=-0.81 \mathrm{~V}, 9.5 \times 8.0 \mathrm{~nm}\right)$. The cyanobiphenyl headgroup appears bright in the STM image relative to the alkyl tail, in accordance with the electron density distribution of the molecule's HOMO (inset). The histogram shows an experimental alignment distribution based on 200 observations from several samples prepared in a $7.2 \mathrm{kG}$ field. The solid line corresponds to the model described in the text. 
above $\sim 2 \mathrm{kG}$. Alignment showed little additional improvement above this threshold, because adsorbates almost always chose the local substrate vector making the row-field angle as close as possible to $\pm 54.6^{\circ}$, while maintaining substrate registry. Films prepared in the absence of a field showed random macroscopic orientational order.

Alignment mechanism. - The 8CB monolayer began to crystallize [7] at $27-29^{\circ} \mathrm{C}$, after the bulk LC had cooled to the smectic- $A$ phase [4]. We estimate the TA monolayer crystallized at $30-40{ }^{\circ} \mathrm{C}$, near the center of ZLI-1565's nematic range, based on the behavior of related aliphatic adsorbates on graphite [8]. Since substrates were immersed in LC solutions at $\sim 100{ }^{\circ} \mathrm{C}$, molecules initially adsorbed in a thermally disordered state (i.e., they did not adsorb prealigned). Furthermore, the orientation of an aligned film could not be altered by placing it back in the field at a different orientation, unless the temperature was raised above the monolayer melting point. Thus molecular alignment originated after adsorption, but during an early stage of film growth, when molecules were forming small crystalline aggregates. Once the number of molecules in an aggregate grew beyond a critical value $N$, aggregate rotation was no longer possible and orientation was fixed (see below).

From these observations, we propose a simple model for communication of orientational order to the developing film. There are three factors influencing adsorbate orientation: (1) interaction with the substrate, (2) direct interaction with the external field, and (3) interaction with the LC fluid. The orientational energy of an adsorbate can be written as a sum of these contributions, $E_{\text {orientation }}(\phi)=$ $E_{\text {substrate }}+E_{\text {field }}+E_{\text {fluid. }}$.

The first term represents adsorbate-substrate interactions, which have by far the strongest influence. At the maximum field strength produced by our magnet (13 kG), molecules in both films remained commensurate with the substrate, and the detailed structure of the unit cell was identical to samples prepared with no field. The magnetic field's influence was therefore limited to breaking the threefold orientational degeneracy of the substrate, causing domains to select the local substrate direction that allowed molecules to most nearly align in the preferred direction.

A calculation of the second term, direct interaction of adsorbed molecules with the external field, showed that it was insignificant. 8CB's biphenyl moiety results in stronger anisotropic interactions with a magnetic field than TA, but these interactions are nonetheless very weak. The energetic cost of misaligning an entire domain of $m$ molecules by $90^{\circ}$ in a field of strength $H$ is $E_{\text {field }}=m w \Delta \chi H^{2} / 2$, where $\Delta \chi$ is the mass magnetic susceptibility anisotropy and $w$ is the molecular weight. The grade of HOPG used in this work resulted in an average adlayer domain size $\sim 1 \mu \mathrm{m}^{2}$, or $m \sim 10^{6}$ molecules, so for $8 \mathrm{CB}, E_{\text {field }} \leq 10^{-2} k T$ per domain in a $7.2 \mathrm{kG}$ field at $28^{\circ} \mathrm{C}$. This energy is at least 2 orders of magnitude too small to account for the statistical excess of favored orientations in Figs. 2 and 3.
It is the third factor-interaction between aggregates of adsorbed molecules and the LC fluid above them - that produces alignment in these films. While surface-induced ordering of LCs is a well-known and technologically important phenomenon, the reverse process, i.e., LCinduced surface ordering, has not to our knowledge been previously described. As is the case for surface-induced bulk alignment, we believe adlayer alignment arises from the LC's anisotropic surface tension, which causes the director to adopt a preferred orientation ("anchor") at an interface [9]. In our systems, the LCs underwent planar anchoring, meaning the director oriented parallel to the surface, along a preferred azimuthal direction [10]. If an aggregate's easy axis does not coincide with the bulk director orientation imposed by the field, the free energy of the system (aggregate + interfacial fluid) increases by an amount proportional to this mismatch. Although the field strengths used here had little direct influence on monolayer alignment, they were sufficient to align the bulk solvent, where macroscopic numbers of molecules act collectively [11]. We confirmed this using polarizing optical microscopy. The following treatment of these anisotropic fluid-adlayer interactions qualitatively accounts for the observed alignment phenomena.

In a nematic solvent, excess free energy arises primarily from a twist distortion in the director field above misaligned aggregates. By twisting, the LC balances the alignment torque exerted by the field with restoring torque from curvature elasticity and surface anchoring. The twist distortion extends a distance $d=H^{-1} \sqrt{K_{22} / \Delta \chi} \sim$ $1-10 \mu \mathrm{m}$ into the bulk, after which the director orients parallel to the field [9]. Here $K_{22}$ is the twist elastic constant. Patrick et al. have shown that interdomain orientational interactions in $8 \mathrm{CB}$ films mediated by an interfacial LC fluid extend less than $0.25 \mu \mathrm{m}$ laterally, which is less than the average terrace size [12]. Thus we consider only isolated aggregates. In the limit of strong anchoring (anchoring energy $\gg H \sqrt{K_{22} \Delta \chi} \sim 10^{-6} \mathrm{~J} \mathrm{~m}^{-2}$ ), the elastic torque exerted on each aggregate by the solvent has a particularly simple form: $\tau=-N a H \sqrt{K_{22} \Delta \chi} \sin \left(\phi_{0}-\phi\right)$, where $a$ is the area per molecule in the crystalline film [13]. If the condition for strong anchoring is not satisfied, the director rotates at the interface, somewhat reducing the torque exerted on the aggregate. The free energy cost of aggregate misalignment is found by integrating the torque through the misalignment angle: $E_{\text {fluid }}^{\text {nematic }}=-N a H \sqrt{K_{22} \Delta \chi} \cos \left(\phi_{0}-\phi\right)$, where for TA, $\phi_{0}=12^{\circ}$.

In a smectic solvent, twist distortions are formally disallowed due to interlayer incompressibility. Therefore in the limit of high field strength, the director remains approximately parallel to the external field right down to the surface. In this case, the excess free energy of aggregate misalignment arises from a mismatch between the director orientation at the surface and the aggregate's anchoring direction. This energy is often modeled with the 
Rapini-Papoular potential [14]: $\quad E_{\text {fluid }}^{\text {smectic }}=\mathrm{NaW} /$ $2 \sin ^{2}\left(\phi_{0}-\phi\right)$, where $W$ is the anchoring energy, and for $8 \mathrm{CB}, \phi_{0}= \pm 54.6^{\circ}$.

Using these expressions for $E_{\text {fluid, the orienta- }}$ tional distribution of an ensemble of aggregates can be calculated if one assumes the film develops in thermal equilibrium (see below). The probability $P(\phi)=Q^{-1} \exp \left[-E_{\text {fluid }}(\phi) / k T\right]$, where $T$ is the temperature of monolayer formation, the partition function $Q=\sum_{\phi} \exp \left[-E_{\mathrm{fluid}}(\phi) / k T\right], k$ is Boltzmann's constant, and $\phi$ is restricted to orientations consistent with local substrate registry due to overwhelming adsorbate-substrate interactions.

We used these expressions to separately fit the distributions in Figs. 2 and 3 to find the aggregate size $N$ that described each system best. For the anchoring energy of $8 \mathrm{CB}$, we chose $W=5 \times 10^{-5} \mathrm{~J} \mathrm{~m}^{-2}$, a typical value on crystalline surfaces. The fitting procedure yielded $N_{\mathrm{TA}}=$ $2 \pm 2 \times 10^{3}$ and $N_{8 \mathrm{CB}}=6 \pm 7 \times 10^{2}$ molecules, with the calculated distributions shown as solid lines in Figs. 2 and 3. We note that $N_{8 \mathrm{CB}}$ agrees well with the nucleus size reported in Ref. [7].

The single-molecule alignment energy $E_{\text {fluid }} / N \ll k T$ at the crystallization temperature. Therefore, only adsorbates that are members of a crystalline aggregate - and hence act collectively - can possess significant uniaxial alignment. We also observe that the aggregate size $N$ is much smaller than the average domain size $m$ in the fully developed film, the latter being of the order $m \sim 10^{6}$ molecules. This is consistent with the observation that film orientation cannot be altered by replacing an aligned sample in the field at a different orientation; the aggregates have grown too large to rotate. Rotation presents an energetic barrier that increases rapidly with aggregate size, because it entails a transition through an incommensurate state. The orientational statistics measured from fully developed films thus reflect the alignment distribution during the early stages of film formation, since large aggregates cannot overcome the rotational activation barrier on experimental time scales.

Even in the strongest field, the maximum energy cost of aggregate misalignment is only a small fraction of the thermodynamic driving force for nucleation. With $8 \mathrm{CB}$, for example, we calculate the ratio of these two energies to be $\sim 0.1 \%$ at $28^{\circ} \mathrm{C}$ [7]. This explains why the detailed arrangement of molecules within the unit cell is unchanged from films prepared outside the field: The field selects among degenerate aggregate orientations, but does not significantly perturb adsorbate-substrate or adsorbateadsorbate interactions.

It has been known since the early 19th century that if one solid crystal is grown in contact with another solid crystal of different chemical constitution, the growing crystal can be oriented through this contact [15]. Our findings show for the first time that this principle also applies to solid crystals grown in contact with thermotropic liquid crystals. The advantage of LC alignment media is that orientation can be controlled by an external field. Because alignment arises from adsorbate-LC fluid interactions - rather than from direct interaction between the adsorbate and the field - it may be possible to prepare uniaxial films from a variety of different building blocks deposited as layer(s) from a LC solvent or suspension.

This work was supported by the National Science Foundation (CHE-9714140) and a Cottrell College Science Award from Research Corporation (CC4564).

*To whom correspondence should be addressed.

Email address: patrick@chem.wwu.edu

[1] See, for instance, G.R. Desiraju, Crystal Engineering - The Design of Organic Solids (Elsevier, New York, 1989); A.C. Hillier and M. D. Ward, Science 263, 1261 (1994).

[2] Structure and Properties of Molecular Crystals, edited by M. Pierrot (Elsevier, Amsterdam, 1990).

[3] Merck KGaA, Darmstadt, Germany. $T_{C \rightarrow N}<-40{ }^{\circ} \mathrm{C}$, $T_{N \rightarrow I}=85^{\circ} \mathrm{C}, \Delta \chi=6.3 \times 10^{-8} \mathrm{~cm}^{3} \mathrm{~g}^{-1}, K_{22}=6.9 \times$ $10^{-7}$ dyn. Physical constants are those reported by the manufacturer.

[4] $T_{C \rightarrow \text { Sm-A }}=22^{\circ} \mathrm{C}, \quad T_{\text {Sm-A } \rightarrow N}=33^{\circ} \mathrm{C}, \quad T_{N \rightarrow I}=41^{\circ} \mathrm{C}$, and $\Delta_{\chi} \sim 1 \times 10^{-7} \mathrm{cgs}$ units [Special supplement on "Sources of thermodynamic data on mesogens," edited by A. Beguin et al. [Mol. Cryst. Liq. Cryst. 115, 1 (1984)]; N. V. Madhusudana and R. Pratibha, Mol. Cryst. Liq. Cryst. 89, 249 (1979)]. These transition temperatures are not significantly affected by a magnetic field [C. R. Rosenblatt, Phys. Rev. A 24, 2236 (1981)].

[5] J. S. Foster and J. E. Frommer, Nature (London) 333, 542 (1988).

[6] J. P. Rabe and S. Buchholz, Science 253, 424 (1991).

[7] D. L. Patrick, V. J. Cee, and T. P. Beebe, Jr., J. Phys. Chem. 100, 8478 (1996).

[8] L. Askadskaya and J. P. Rabe, Phys. Rev. Lett. 69, 1395 (1992).

[9] A. A. Sonin, The Surface Physics of Liquid Crystals (Gordon and Breach, Luxembourg, 1995).

[10] Polarized optical microscopy was used to confirm that the LC director in the bulk fluid orients parallel to the field and in the plane of the substrate in both systems.

[11] A. J. Dekker, Can. J. Phys. 65, 1185 (1987).

[12] D. L. Patrick, V. J. Cee, M. D. Morse, and T. P. Beebe, Jr., J. Phys. Chem. B 103, 8328 (1999).

[13] S. Faetti, M. Gatti, and V. Palleschi, Rev. Phys. Appl. 21, 451 (1986).

[14] A. Rapini and M. J. Papoular, J. Phys. (Paris), Colloq. 30, C4-54 (1969).

[15] M. L. Frankenheim, Pogg. Ann. 37, 516 (1836). 